\title{
A preliminary stochastic analysis of the uncertainty of natural processes related to renewable energy resources
}

\author{
Elli Klousakou, Maria Chalakatevaki, Panayiotis Dimitriadis, Theano Iliopoulou, Romanos Ioannidis, \\ Georgios Karakatsanis, Andreas Efstratiadis, Nikos Mamasis, Romina Tomani, Efthimis Chardavellas, and \\ Demetris Koutsoyiannis
}

Department of Water Resources, National Technical University of Athens, 15780 Zografou, Greece

Correspondence: Elli Klousakou (e.klousakou@gmail.com)

Received: 31 May 2018 - Revised: 21 July 2018 - Accepted: 30 July 2018 - Published: 22 August 2018

\begin{abstract}
The ever-increasing energy demand has led to overexploitation of fossil fuels deposits, while renewables offer a viable alternative. Since renewable energy resources derive from phenomena related to either atmospheric or geophysical processes, unpredictability is inherent to renewable energy systems. An innovative and simple stochastic tool, the climacogram, was chosen to explore the degree of unpredictability. By applying the climacogram across the related timeseries and spatial-series it was feasible to identify the degree of unpredictability in each process through the Hurst parameter, an index that quantifies the level of uncertainty. All examined processes display a Hurst parameter larger than 0.5 , indicating increased uncertainty on the long term. This implies that only through stochastic analysis may renewable energy resources be reliably manageable and cost efficient. In this context, a pilot application of a hybrid renewable energy system in the Greek island of Astypalaia is discussed, for which we show how the uncertainty (in terms of variability) of the input hydrometeorological processes alters the uncertainty of the output energy values.
\end{abstract}

\section{Introduction}

Nowadays fossil fuels cover $80 \%$ of the energy demand globally. However, over the past 20 years, renewable energy resources (with the most notable being hydropower, ocean, wind and solar energy) have gradually become widely accepted due to the need for achieving energy security and the global increase in environmental sensitivity. Yet, the irregular variability of the related geophysical processes introduces a remarkable degree of uncertainty to the renew- able energy resources systems and, thus, the predictability of related energy production is often limited. Specifically, the more complex the process (in terms of stochastic behavior, Koutsoyiannis, 2017) the larger the introduced uncertainty, even for the conventional energy sources. For example, although it is expected that the energy produced by fossil fuels is not varying significantly in time, suggesting that it should follow a deterministic behaviour (and thus fully predictable in all scales and time horizons) due to fixed deposits, its production, at least in the long term, is in fact uncertain, due to monetary, currency (e.g. petro-currency) and geopolitical reasons (Karakatsanis et al., 2017). However, the great challenge with renewables is the lack of predictability at all temporal scales, thus affecting both the design and operation of the associated systems.

It is noted that a Hurst-Kolmogorov (HK) behaviour (indicating long-term persistence across scales) is expected in all geophysical and hydrometeorological processes (Koutsoyiannis, 2017). Interestingly, it has been recently shown (Dimitriadis, 2017) that processes with completely different nature such as the different hydrometeorological processes (or even experimental turbulence (Dimitiadis, 2017; Chardavellas et al., 2018; references therein) exhibit certain stochastic similarities (e.g., long-term persistence), revealing a common stochastic nature. Therefore, a generic and parsimonious model such as the HK-type can unify our view on such processes that have a completely different deterministic behaviour but a common stochastic one (Koutsoyiannis, 2016). Although uncertainty rules most natural processes, natural systems instill both randomness and determinism in their processes. The prevailing understanding that unpredictability is a component of predictability, sug- 


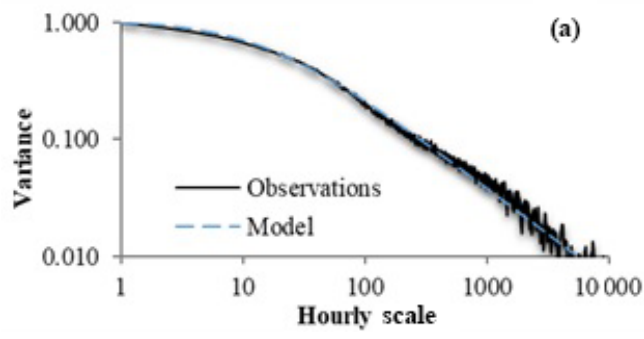

Figure 1. Wind speed climacogram (a) and precipitation (b).

gests that there is a "virus of randomness" infecting only specific phenomena. However recent studies indicate that this hypothesis is inaccurate and misleading (Dimitriadis et al., 2016); in fact, determinism and unpredictability coexist, in which predictability dominates on short time-windows and unpredictability on wider ones,

The term energy sources is directly related to natural resources, geophysical and weather phenomena that are governed by inherent uncertainty, and therefore, unpredictability. This uncertainty strongly affects the management and operation of energy systems as well as the cost and sustainability of investments in the energy sector. On the other hand, energy demand is directly correlated to financial, environmental and societal factors (Tyralis et al., 2017b). Therefore, satisfying energy demand, or else achieving energy security, in a reliable and sustainable manner is a matter of great societal, financial and environmental importance, and it is considered one of the main technological goals of our era.

To this end, the robust characterization of the predictability of the natural processes related to renewable energy production, or equivalently, of the related uncertainty, is a key first step for the design and management of energy systems, and it may also inform more efficient and reliable investments in the energy sector (while as a consequence energy could be produced in a more reliable manner). For the purpose of quantifying multi-scale uncertainty, this study inspects the variability of natural processes directly linked to renewable energy management by means of a parsimonious stochastic model able of revealing the second-order properties of timeseries (Klousakou et al., 2018).

\section{Methodology: Uncertainty quantification based on the second-order dependence structure}

A robust measure of the inherent uncertainty of the introduced data is the Hurst parameter (Dimitriadis and Koutsoyiannis, 2015). There are various methods to estimate the Hurst parameter, based on the climacogram, the power spectrum, the autocovariance, etc. (Dimitriadis and Koutsoyiannis, 2015); the climacogram is defined as the log-log plot of variance of the averaged process versus averaging time scale,

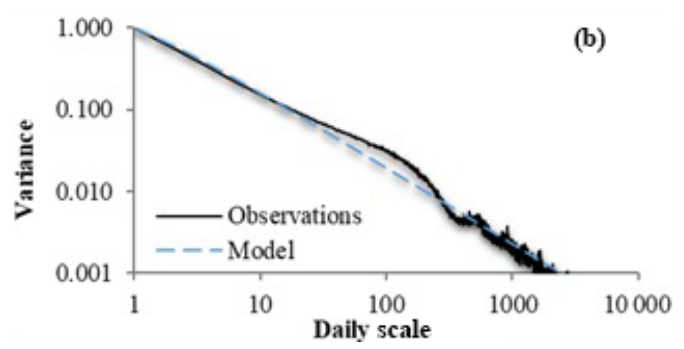

whereas the power spectrum of a random process describes the distribution of its variance over the frequency.

Scientific studies (Dimitriadis, 2017) have shown that the climacogram is a more valid method (Dimitriadis and Koutsoyiannis, 2015), among the three, for estimating the Hurst parameter and so, it is very useful in detecting long term change or in general the dependence across scale. As far as the power spectrum or autocovariance is concerned, their estimation from data may distort the true behaviour of the processes and thus, may lead to false interpretation. Furthermore, the climacogram has the smallest estimation error compared to the other two methods (with the power spectrum presenting the largest one) and a simple expression for statistical bias. The autocovariance and power spectrum are also prone to discretization errors as their values can never be equal with the true value in continuous time. Finally, the power spectrum has a complicated definition (based on the Fourier transformation of the autocovariance), which involves computational cost. Therefore, the climacogram can calculate the Hurst parameter more reliably.

In order to conduct the current research, the methodology described below was used to calculate the degree of uncertainty in the examined processes. After the collection of longtimeseries from different global regions in order to compile timeseries in various time-scales (monthly, daily, hourly and semi-hourly time scale), a flexible climacogram-based stochastic model was applied in each sample in order to estimate the degree of the induced uncertainty of the parent processes:

$\gamma(k)=\gamma(1)((1+1 / q) /(1+k / q))^{(2-2 H)}$

where $\gamma$ is the standardized climacogram, $k$ the time scale (here represented as an integral multiple of the finest available time scale), $H$ is the Hurst parameter, and $q$ is a scaleparameter.

\section{Uncertainty in processes related to renewable energy resources (solar, wind, marine energy and hydropower)}

Due to the effect of the statistical bias, for a robust estimation of the Hurst parameter, we require either a single long-length 

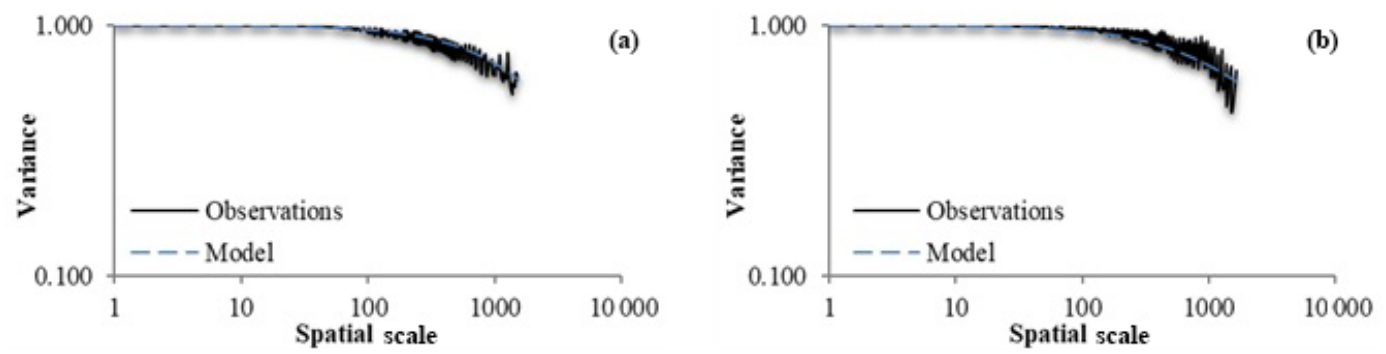

Figure 2. Climacograms for temperature borehole data at two different times.
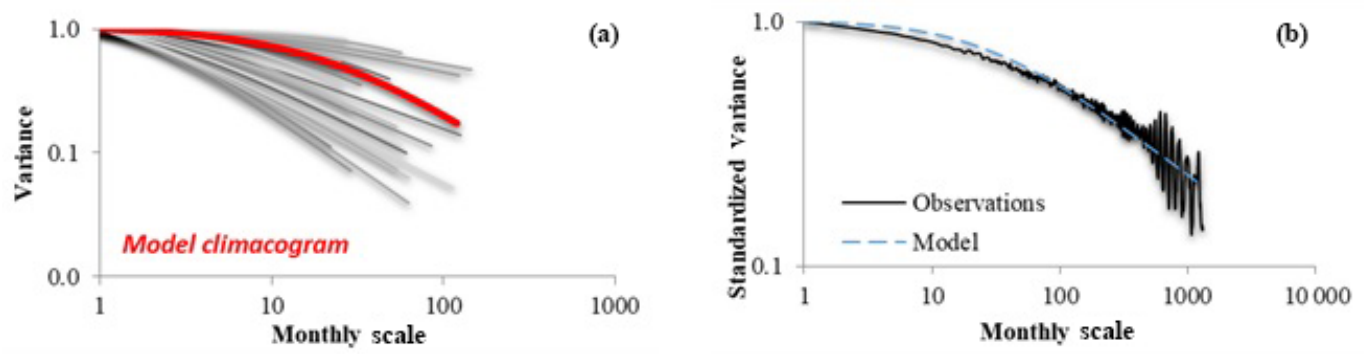

Figure 3. Climacogram for tides (a) and currents (b).

timeseries or multiple shorter timeseries in case where the former is limited.

In terms of the solar energy, renewable energy can be produced from solar radiation using solar panels. Related timeseries were collected from multiple stations located in the United States of America. It is noted that the climacogram of solar radiation timeseries depicts double periodicity (caused by the diurnal and seasonal cycles), which can be dealt with double cyclo-stationary models (Koudouris et al., 2017). For this process, the Hurst parameter was estimated at 0.81 . Additionally, for an illustration of the uncertainty in the wind speed process (related to the wind energy), the climacogram was estimated for an hourly wind station (Gkolemis et al., 2018; where the related data were obtained from), with a Hurst parameter equal to 0.61 (Fig. 1). Finally, the climacogram was applied to precipitation (related to hydropower; the oldest form of renewable energy) (Chalakatevaki et al., 2018; where the related data were obtained from), and the Hurst parameter was estimated at 0.54 (Fig. 1).

The exploitation of geothermal energy began in the early 20th century. Up to now, its use has been extended because of the increase of the energy demand. In order to investigate the uncertainty for this process, temperature data were collected from a borehole in Idaho, USA (https://openei.org/ doe-opendata/, last access: 19 August 2018). Two different measurements (one in 17 January 2012 and the other on 21 January 2012) provided spatial-series in order to define the Hurst parameter related to geothermal power. In both measurements (Fig. 2) the Hurst parameter was estimated 0.75 .
An alternative but not extensively applied form of renewable energy is the ocean (or else marine) energy. Its components can be classified into two categories (Edenhofer et al., 2012). The first category includes tides, waves and currents which are generated by gravitational forces. As far as tides are concerned, 22 stations across the state of Florida in the USA (https://tidesandcurrents.noaa.gov/products.html, last access: 19 August 2018) provided an adequate number of timeseries to define the Hurst parameter, which varied from 0.50 to 0.87 among the different stations and was $0.60 \mathrm{on}$ average (Fig. 3). Data related to currents, were gathered once again from the state of Florida, and the Hurst parameter was found 0.80 (Fig. 3).

In order to investigate the stochastic behaviour of waves, four different variables were examined from a station in Hilo (Hawaii; http://www.pacioos.hawaii.edu/waves/ buoy-hilo/, last access: 19 August 2018): height, direction, mean period and peak period. The Hurst parameter was calculated 0.75 for height, 0.87 for direction, 0.84 for peak period and 0.66 for mean period (Fig. 4).

The second category of marine energy is expressed through sources related to thermal processes. Specifically, energy can be produced from temperature differences either from warm surface water $\left(22-27^{\circ} \mathrm{C}\right)$ or very cold water $\left(4-7^{\circ} \mathrm{C}\right)$ at a depth of approximately $1 \mathrm{~km}$; this form of energy is called ocean thermal energy (Eternadi et al., 2011). All the necessary data were acquired from two different stations located in Florida (SSW28", DSW40"; https://catalog.data.gov/dataset/temperature-salinity, last access: 19 August 2018). Respectively, in both stations the Hurst parameter was estimated close to 0.55 (Fig. 5). 

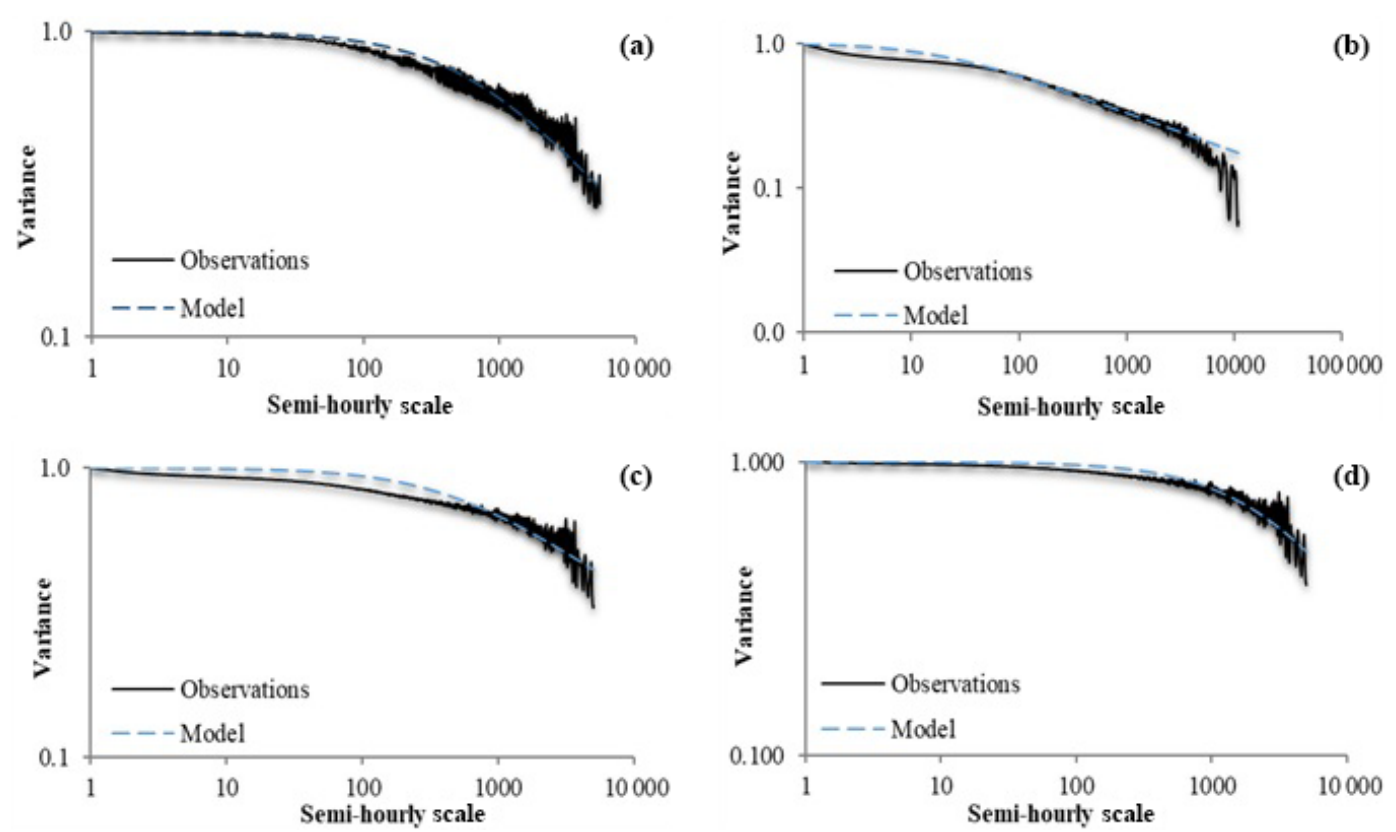

Figure 4. Climacogram for waves' height (a), direction (b), peak period (c) and mean period (d) in Hilo, Hawaii.
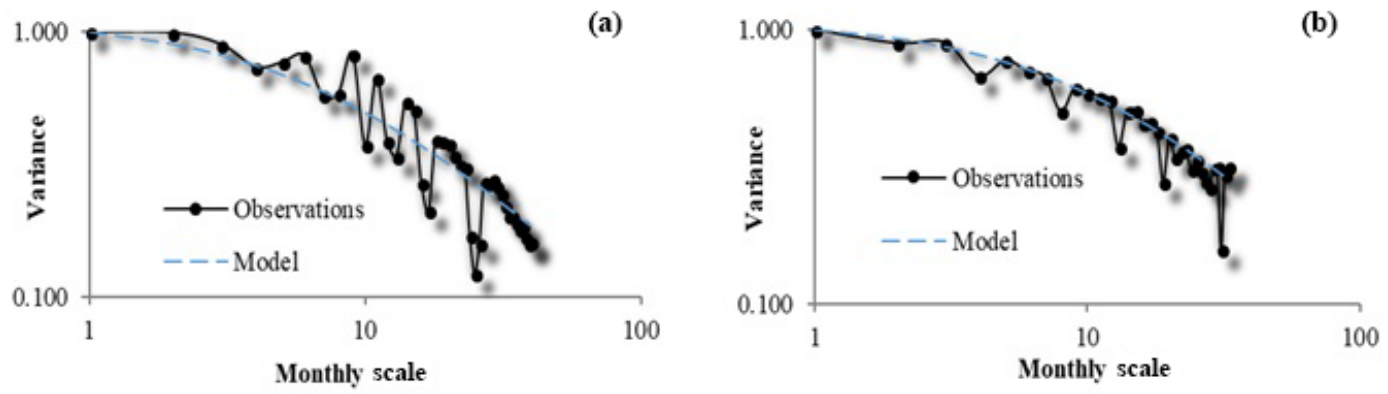

Figure 5. Climacogram for water temperature in two different stations in Florida (SSW28" and DSW40" respectively).

Moreover, salinity differences can also generate renewable energy. Salinity Gradient Energy (SGE) (Schaetzle and Buisman, 2015), often known as the blue energy, relies on the energy that is released when two solutions with different salinities come in contact and mix. The same database used for temperature timeseries was also used in the case of salinity. In both climacograms (each one referring to the respective stations like the temperature data in Fig. 5) the Hurst parameter was found again 0.55 (Fig. 6).

Although marine energy is a very promising energy source, it has limited application; this also justifies the limited research made so far on the uncertainty of the related processes. Nevertheless, the marine energy is expected to have a significant contribution in the near future, with a theoretical potential energy production estimated at $7500 \mathrm{EJ} \mathrm{yr}^{-1}$ (Edenhofer et al., 2012).

\section{The significance of uncertainty for the green energy autonomy in non-connected islands}

Many islands around the world, are not connected to the electricity network of the mainland and depend only on oilfueled power plants, which have a high oil import cost and a high environmental impact. The continuous advances in renewable energy technology along with the gradual reduction of installation costs, pave the way towards a wider utilization of renewable energy. Effective planning of a renewable energy resource system requires the investigation of renewable resources and energy demand. Such a case study was implemented in a small non-connected Greek island located in the Aegean Sea, named Astypalaia, where several renewable resources were examined, focusing on hydropower, solar, marine and wind energy. The hydrometeorological variables were treated as stochastic processes. In the absence of meteorological stations at the exact location, the diurnal and seasonal periodicity of temperature and dew point were es- 

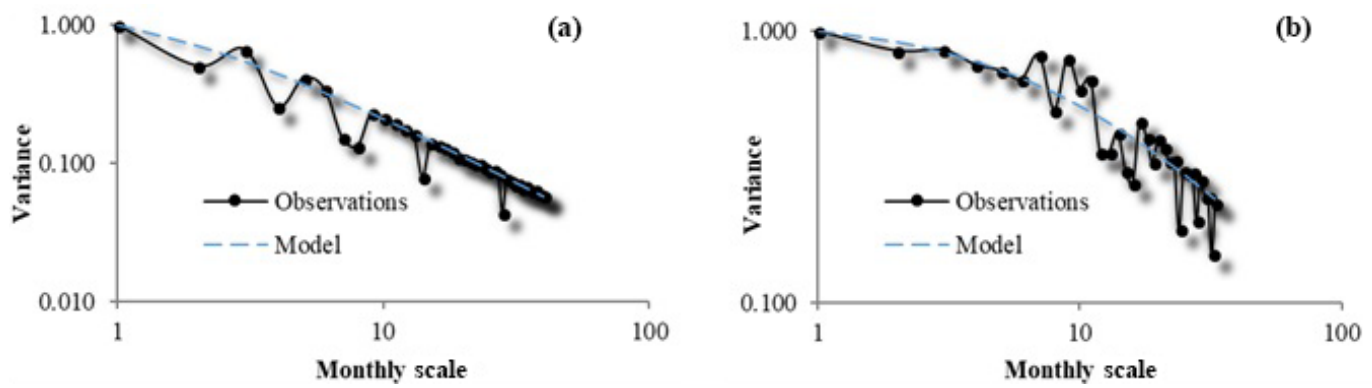

Figure 6. Climacogram for salinities in two different stations in Florida (SSW28" and DSW40" respectively).
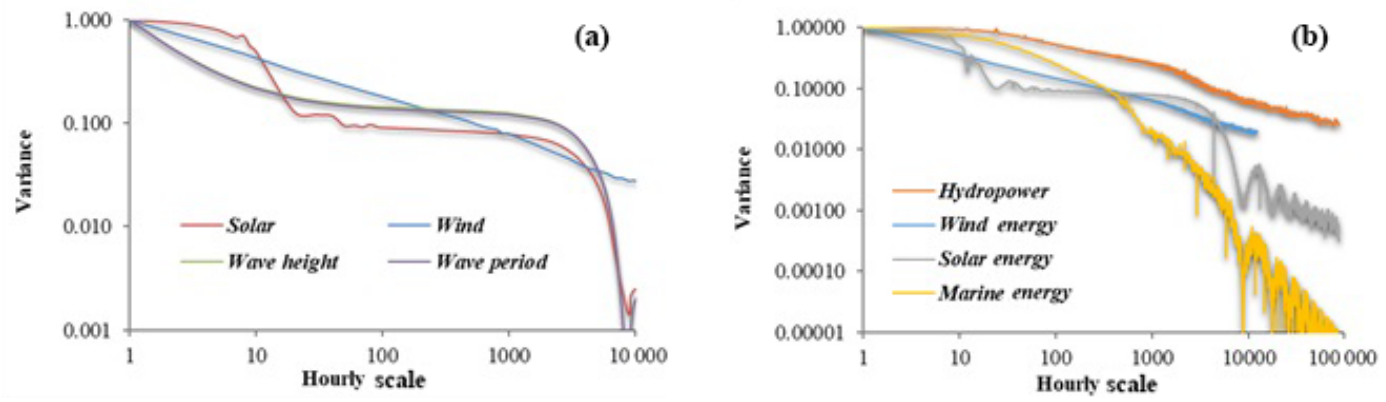

Figure 7. Climacograms demonstrating the uncertainty among hydrometeorological processes (a) and the various renewable energy technologies (b) investigated in the case study of Astypalaia Island.

timated from nearby stations (similar has been done in the analysis by Hadjimitsis et al., 2017), as well as the energy demand and the cross-correlations among all processes (similar to what has been done in the analysis by Koskinas et al., 2017). Moreover, the energy demand data were produced through synthetic timeseries due to limited data. Each synthetic timeseries was simulated from specific stochastic models aiming to maintain the structure and the characteristics of the real timeseries from which they were derived.

By applying the climacogram among all the related timeseries referring to Astypalaia, it is now obvious that uncertainty exists not only in the relevant natural processes but in the whole energy system as well. A significant degree of uncertainty is introduced in the energy system through means of energy production due to technical failures of the machines (which can delay or modify the energy production time schedule). Thus, in order to define the degree of unpredictability in each part of the energy system, it is necessary to include all sources of uncertainty. Furthermore, there are noticeable differences in the degree of uncertainty (different slope in the climacogram) among the various processes and energy systems. Consequently, optimization plans, based on stochastic analysis, are of great importance for the energy system in order to render it manageable, despite unpredictability.

A pilot application in the island of Astypalaia indicates that by creating an energy mix through stochastic investigation, including all the aforementioned renewable energy sources, in combination with the fuel oil could cover efficiently the energy demand of the island (Chalakatevaki et al., 2017). However, taking into consideration that none of the aforementioned energy sources has a constant and adequate reservoir, another alternative solution could be applied. The addition of a geothermal and biomass system to the energy balance could cover all energy requirements effectively. The latter energy source would be easily controlled by operators and provide energy at all timescales.

The possibility of hydropower production in the island was also studied and a simulation framework for small-scale reservoir systems was modelled Papoulakos et al., 2017), in association with high solar (Koudouris et al., 2017), wind and wave energy (Moschos et al., 2017). The potential of deploying agricultural residues, as well as cultivating energy crops with low irrigation demands for biomass energy production, was investigated. Taking into account that Astypalaia is located at the Volcanic Arc of southern Aegean Sea the potential of implementing measurements in order to verify the possibility of geothermal energy was also considered (Chalakatevaki et al., 2017).

It is noted that when designing an energy system based only on weather-related renewable energy resources, i.e. solar, wind, marine and hydroelectric energy, the peak hourly demand is not satisfied and a great amount of energy surplus, uncontrollable and unsynchronized with the demand, is produced. Therefore, biomass and geothermal resources were added to the energy mix in order to cover the remain- 
ing deficit and a pumped-storage system was used to store electric energy surplus and satisfy the peak deficits (Chalakatevaki et al., 2017). The implementation of the renewable energy mix resulted in a high installed capacity (since the installed power of each renewable does not always synchronize with the demand) and a rather high installation cost. The examined solutions still had a high implementation and maintenance cost, and therefore, it was reasonable to consider further development of thermal stations. In the future, it is expected that the cost of renewable resources will be further reduced and the proposed solutions could be more attractive from a financial, societal as well as environmental point of view.

\section{Discussion}

The primary purpose of this research is to define the degree of uncertainty in geosciences and human systems with contribution to sociohydrology. By applying the climacogram, the Hurst exponent was calculated, allowing us to quantify the degree of unpredictability. The Hurst parameter varies across different time scales, which is also supported by recent research results (Dimitriadis, 2017) and it is estimated greater than 0.5 (even using a downward biased estimator through the climacogram) for all examined processes. Consequently, several natural processes (marine, precipitation, sun and wind) exhibit the Hurst-Kolmogorov behaviour and not a Markovian or a white noise one. Through the climacogrambased analysis it is revealed that in long time scales, the larger the Hurst parameter the shorter the predictability window while in small time-scales the larger the correlation parameter the lower the degree of uncertainty. Since the Hurst parameter is greater than 0.5 , the examined processes associated with renewable energy resources systems are governed by a great degree of uncertainty. Yet the degree of uncertainty significantly varies across the examined processes. Specifically, we find solar radiation and wave height to exhibit large Hurst parameters, while others such as precipitation exhibit relatively lower ones, the latter being consistent with findings in (Iliopoulou et al., 2016; Tyralis et al., 2017a). The degree of variability of the different processes at the time-scales of interest are crucial for the design and operation of a renewable energy system, where the operation rules of each energy source should be specified in a way that ensures reliability and optimal performance of the whole system. For example, a general operational rule of such systems is that energy from the least reliable sources, e.g. wind energy, is consumed in priority.

Recent analyses concerning the renewable energy design and management for the non-connected island of Astypalaia have illustrated how the uncertainty of several renewable energy sources can be efficiently managed through stochastic analysis (Chalakatevaki et al., 2017; Papoulakos et al., 2017). Therefore, stochastic analysis is essential in the renewable energy management both for the analysis of predictability of the related natural processes and for the analysis of the system dynamics and optimal design and operation under increased uncertainty. Yet the stochastic aspect of renewable energy systems still remains a relatively underexplored field. Further research will focus on the exploration of the uncertainty of the related processes based on more extended datasets as well as on the quantification of the propagation of uncertainty from the natural processes to the final energy production.

Data availability. All databases from which the data related to Marine Energy and Geothermal Energy were retrieved, can be found inside the text in the form of links. The datasets used in any other case are available from the corresponding author on reasonable request.

Author contributions. PD and DK conceived of the initial idea and EK undertook its realization through the presented computational study which was based on DK's climacogram concept. DK, PD and IT supervised the findings of this work. EK took the lead in writing the manuscript. All authors provided critical feedback and helped shape the research, analysis and manuscript.

Competing interests. The authors declare that they have no conflict of interest.

Special issue statement. This article is part of the special issue "European Geosciences Union General Assembly 2018, EGU Division Energy, Resources \& Environment (ERE)". It is a result of the EGU General Assembly 2018, Vienna, Austria, 8-13 April 2018.

Acknowledgements. The authors thank warmly both Alberto Montanari and an anonymous reviewer for their constructive comments which helped us improve the paper.

Edited by: Sonja Martens

Reviewed by: Alberto Montanari and one anonymous referee

\section{References}

Chalakatevaki, M., Stamou, P., Karali, S., Daniil, V., Dimitriadis, P. Tzouka, K., Iliopoulou, T., Koutsoyiannis, D., Papanicolaou P., and Mamassis, N.: Creating the electric energy mix in a non-connected island, Energy Proced., 125, 425-434, https://doi.org/10.1016/j.egypro.2017.08.089, 2017.

Chalakatevaki, M., Klousakou, E., Dimitriadis, P., Iliopoulou, T., and Koutsoyiannis, D.: Stochastic investigation of the uncertainty of hydrometeorological processes by means of the climacogram, EGU General Assembly, Vienna, Austria, 8-13 April 2018, Geophysical Research Abstracts, EGU2018-17714- 
1, available at: https://www.itia.ntua.gr/en/docinfo/1818/ (last access: 19 August 2018), 2018.

Chardavellas, E., Dimitriadis, P., Papakonstantis, I., Koutsoyiannis, D., and Papanicolaou, P.: Stochastic similarities between the microscale of vertical thermal jet and macroscale hydrometeorological processes, EGU General Assembly, Vienna, Austria, 8-13 April 2018, Geophysical Research Abstracts, EGU2018-17803, available at: https://www.itia.ntua.gr/en/docinfo/1822/ (last access: 19 August 2018), 2018.

Dimitriadis, P.: Hurst-Kolmogorov dynamics in hydrometeorological processes and in the microscale of turbulence, $\mathrm{PhD}$ thesis, Department of Water Resources and Environmental Engineering, National Technical University of Athens, Greece, available at: http://dspace.lib.ntua.gr/handle/123456789/45955? locale-attribute=en (last access: 19 August 2018), 2017.

Dimitriadis, P. and Koutsoyiannis, D.: Climacogram versus autocovariance and power spectrum in stochastic modelling for Markovian and Hurst-Kolmogorov processes, Stoch. Env. Res. Risk A, 29, 1649-1669, https://doi.org/10.1007/s00477-015-1023-7, 2015.

Dimitriadis, P., Koutsoyiannis, D., and Tzouka, K.,:Predictability in dice motion: how does it differ from hydrometeorological processes?, Hydrolog. Sci. J., 61, 1611-1622, https://doi.org/10.1080/02626667.2015.1034128, 2016.

Edenhofer, O., Pichs-Madruga, R., Sokona, Y., Seyboth, K., Matschoss, P., Kadner, S., Zwickel, T., Eickemeier, P., Hansen, G., Schlömer, S., and Von Stechow, C. (Eds.): Renewable energy sources and climate change mitigation, Summary for policy makers and technical summary, Special report of the intergovernmental panel on climate change, Edition 2011, IPCC, USA, Cambridge, UK/New York: Cambridge University, available at: https://www.ipcc.ch/pdf/special-reports/srren/SRREN_ FD_SPM_final.pdf (last access: 19 August 2018), 2012.

Etemadi, A., Emdadi, A., AsefAfshar, O., and Emami, Y.: Electricity generation by the ocean thermal Energy, Energy Proced., 12, 936-943, 2011.

Gkolemis, A., Dimitriadis, P., Karakatsanis, G., Iliopoulou, T., and Koutsoyiannis, D.: A stochastic investigation of the intermittent behaviour of wind; application to renewable energy resources management, EGU General Assembly, Vienna, Austria, 8-13 April 2018, Geophysical Research Abstracts, EGU201815979-3, available at: https://www.itia.ntua.gr/en/docinfo/1795/ (last access: 19 August 2018), 2018.

Hadjimitsis, E., Demetriou, E., Sakellari, K., Tyralis, H., Dimitriadis, P., Iliopoulou, T., and Koutsoyiannis, D.: Investigation of the stochastic nature of temperature and humidity for energy management, Geophysical Research Abstracts, EGU General Assembly, Vienna, Austria, 23-28 April 2017, EGU201710164-5, available at: https://www.itia.ntua.gr/en/docinfo/1687/ (last access: 19 August 2018), 2017.

Iliopoulou, T., Papalexiou, S. M., Markonis, Y., and Koutsoyiannis, D.: Revisiting long-range dependence in annual precipitation, J. Hydrol., 556, 891-900, 2016
Karakatsanis, G., Roussis, D., Moustakis, Y., Gournari, P., Parara, I., Dimitriadis, P., and Koutsoyiannis, D.: Energy, variability and weather finance engineering, Energy Proced., 125, 389-397, 2017.

Klousakou, E., Chalakatevaki, M., Tomani, R., Dimitriadis, P., Efstratiadis, A., Iliopoulou, T., Ioannidis, R., Mamassis, N., and Koutsoyiannis, D.: Stochastic investigation of the uncertainty of atmospheric processes related to renewable energy resources, EGU General Assembly, Vienna, Austria, 8-13 April 2018, Geophysical Research Abstracts, EGU2018-16982-2, available at: http://www.itia.ntua.gr/1789/ (last access: 19 August 2018), 2018.

Koskinas, A., Zacharopoulou, E., Pouliasis, G., Engonopoulos, I., Mavroyeoryos, K., Deligiannis, E., Karakatsanis, G., Dimitriadis, P., Iliopoulou, T., Koutsoyiannis, D., and Tyralis, H.: Simulation of electricity demand in a remote island for optimal planning of a hybrid renewable energy system, EGU General Assembly, Vienna, Austria, 23-28 April 2017, Geophysical Research Abstracts, EGU2017-10495-4, https://www.itia.ntua.gr/ en/docinfo/1684/ (last access: 19 August 2018), 2017.

Koudouris, G., Dimitriadis, P., Iliopoulou, T., Mamassis, N., and Koutsoyiannis, D.: Investigation on the stochastic nature of the solar radiation process, Energy Proced., 125, 398-404, 2017.

Koutsoyiannis, D.: Generic and parsimonious stochastic modelling for hydrology and beyond, Hydrolog. Sci. J., 6, 225-244, https://doi.org/10.1080/02626667.2015.1016950, 2016.

Koutsoyiannis, D.: Entropy production in stochastics, Entropy, 19, 581, https://doi.org/10.3390/e19110581, 2017.

Moschos, E., Manou, G., Dimitriadis, P., Afendoulis, V., Koutsoyiannis, D., and Tsoukala, V.: Harnessing wind and wave resources for a Hybrid Renewable Energy System in remote islands: a combined stochastic and deterministic approach, Energy Proced., 125, 415-424, https://doi.org/10.1016/j.egypro.2017.08.084, 2017.

Papoulakos, K., Pollakis, G., Moustakis, Y., Markopoulos, A., Iliopoulou, T., Dimitriadis, P., Koutsoyiannis, D., and Efstratiadis, A.: Simulation of water-energy fluxes through smallscale reservoir systems under limited data availability, Energy Proced., 125, 405-414, 2017.

Schaetzle, O. and Buisman, C. J. N.: Salinity gradients energy: current state and new trends, Engineering, 1, 164-166, https://doi.org/10.15302/j-eng-2015046, 2015.

Tyralis, H., Dimitriadis, P., Koutsoyiannis, D., O’Connell, P., Tzouka, K., and Iliopoulou, T.: On the long-range dependence properties of annual precipitation using a global network of instrumental measurements, Adv. Water Resour., 111, 301-318, 2017a.

Tyralis, H., Karakatsanis, G., Tzouka, K., and Mamassis, N.: Exploratory data analysis of the electrical energy demand in the time domain in Greece, Energy Proced., 134, 902-918, 2017 b. 